\title{
ARTICLE
}

Received 14 Oct 2013 | Accepted 17 Mar 2014 | Published 23 Apr $2014 \quad$ DOl: 10.1038/ncomms4676

\section{Spatially and temporally reconfigurable assembly of colloidal crystals}

Youngri Kim¹, Aayush A. Shah² \& Michael J. Solomon¹,2

The self-assembly of colloidal crystals is important to the production of materials with functional optical, mechanical and conductive properties. Yet, self-assembly methods are limited by their slow kinetics and lack of structural control in space and time. Refinements such as templating and directed assembly partially address the problem, albeit by introducing fixed surface features such as templates or electrodes. A template-free method to reconfigure colloidal crystals simultaneously in three-dimensional space and time would better align work in colloidal assembly with materials applications. Here, we report a photo-induced assembly method that yields regions either filled with colloidal crystals or completely devoid of colloids. The origin of the effect is found to be electrophoresis of colloids generated by photochemistry at an indium tin oxide-coated substrate. Simple optical manipulations are applied to reconfigure these assembly and depletion regions. Thus, the method represents a new kind of template-free, reconfigurable three-dimensional photolithography.

\footnotetext{
${ }^{1}$ Department of Chemical Engineering, University of Michigan, NCRC B10-A151, 2800 Plymouth Rd., Ann Arbor, Michigan 48109, USA. ${ }^{2}$ Macromolecular Science and Engineering, University of Michigan, NCRC B10-A151, 2800 Plymouth Rd., Ann Arbor, Michigan 48109, USA. Correspondence and requests for materials should be addressed to M.J.S. (email: mjsolo@umich.edu).
} 
$\mathrm{R}$ ecent efforts to produce reconfigurable colloidal assemblies have included holographic optical tweezers ${ }^{1,2}$, optically tunable electrophoretic and electrokinetic assemblies ${ }^{3,4}$, photoresponsive colloids ${ }^{5}$ and DNA-directed assembly ${ }^{6}$. While powerful, these methods, respectively, are limited to small scales, require external electric fields, are not easily reversible, or have to date only yielded two-dimensional patterning. More useful for applications such as adaptive optics ${ }^{7,8}$ or reconfigurable circuit elements ${ }^{9}$ with conductive functionalities ${ }^{10}$ would be an assembly method in which a three-dimensional colloidal crystal could not only be switched on and off in time, but could also be simultaneously controlled in space, on the micron scale, without the restriction of fixed surface features such as electrodes ${ }^{11-13}$ and templates ${ }^{14-16}$.

Light is an effective means for control of colloidal crystals in space and time, because its propagation is easily manipulated by commonly available microscope optics. The effect of light on colloids is typically due to radiation pressure, especially for a system with a mismatch between particle and solvent refractive index ${ }^{17}$. Light-induced assemblies have also been produced at the nanoscale for the specific case of cadmium telluride ${ }^{18}$ and gold ${ }^{19}$ nanoparticles. However, light can also be used to control the rate of chemical reactions, and therefore induce localized ion flows due to a spatial concentration gradient of reactants ${ }^{20}$. These ion flows create non-local electric fields that could electrophoretically assemble colloids.

Here, we show results in which this conceptual idea is implemented. We induce colloidal crystallization in spatially selective regions by using light to generate a local electric field.
The local electric field directs colloidal motion by coupling to charge on the particle, thereby inducing electrophoresis that leads to colloidal assembly. The local electric field is generated by illuminating an indium tin-oxide (ITO)-coated glass substrate at the base of a colloidal suspension with a $488 \mathrm{~nm}$ laser or an ultraviolet (UV) source. Localized illumination of the ITO coating results in motion of the particles either towards or away from the illuminated region. The assembly kinetics and structures are characterized in situ by direct visualization with a confocal laser scanning microscope. The method is fully reconfigurable, noninvasive, operates at low powers and generates structures of complex shape with sharp features.

\section{Results}

Photo-induced colloidal assembly. The system studied, $1.4 \mu \mathrm{m}$ colloidal poly(methyl methacrylate) (PMMA) stabilized with poly(12-hydroxy-stearic acid) (PHSA) and dispersed in a refractive index- and density-matched solvent of cyclohexyl-bromide (CHB) and decahydronaphthalene (decalin), is a common one for colloidal self-assembly ${ }^{21-23}$. A $200 \mu \mathrm{l}$ solution of these colloidal particles, with $50 \mu \mathrm{M}$ of the disassociating species tetrabutylammonium bromide (TBAB) added, was pipetted into a glass vial. The interior of the bottom of the vial had been coated with a $65 \mathrm{~nm}$ thick layer of indium tin oxide (ITO). When a $25 \times 25 \mu \mathrm{m}^{2}$ region of the surface was illuminated with a $488 \mathrm{~nm}$ laser $\left(12 \mathrm{~W} \mathrm{~cm}^{-2}\right)$, accumulation of particles was observed in the vicinity of the illuminated region in about $60 \mathrm{~s}$ (Fig. 1a-c). At this condition, as in many others, the accumulation of particles was a

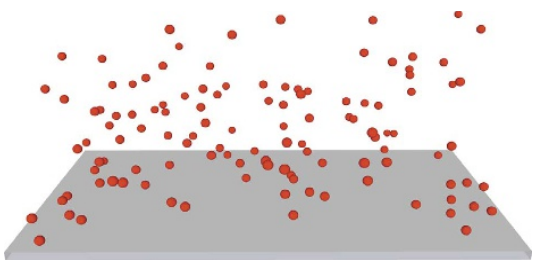

b

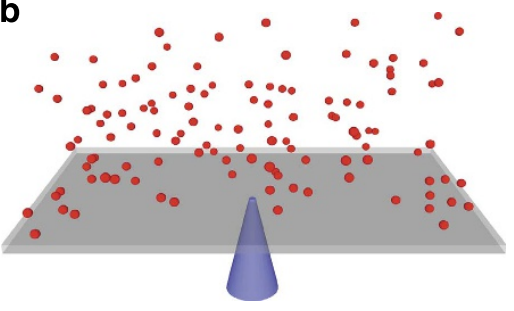

C

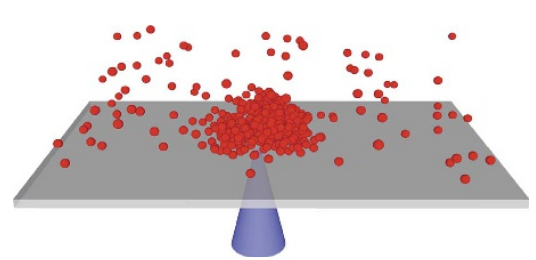

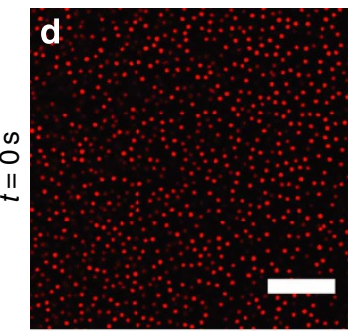
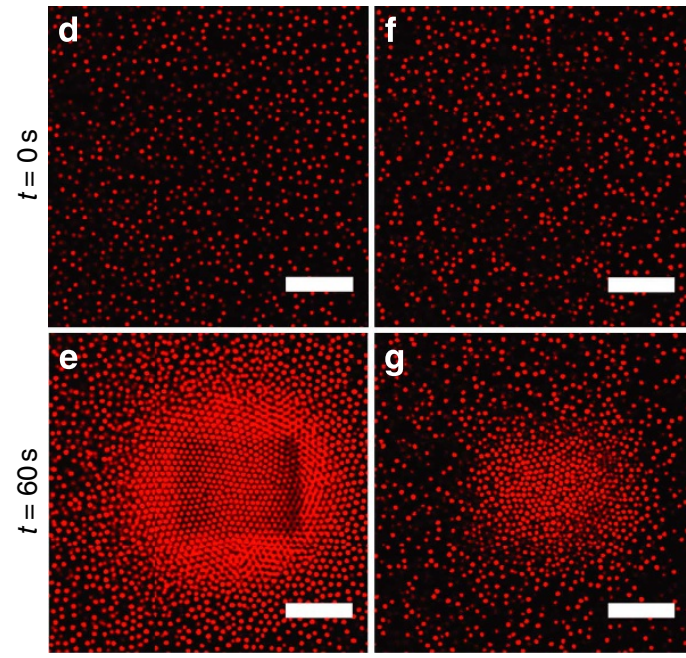
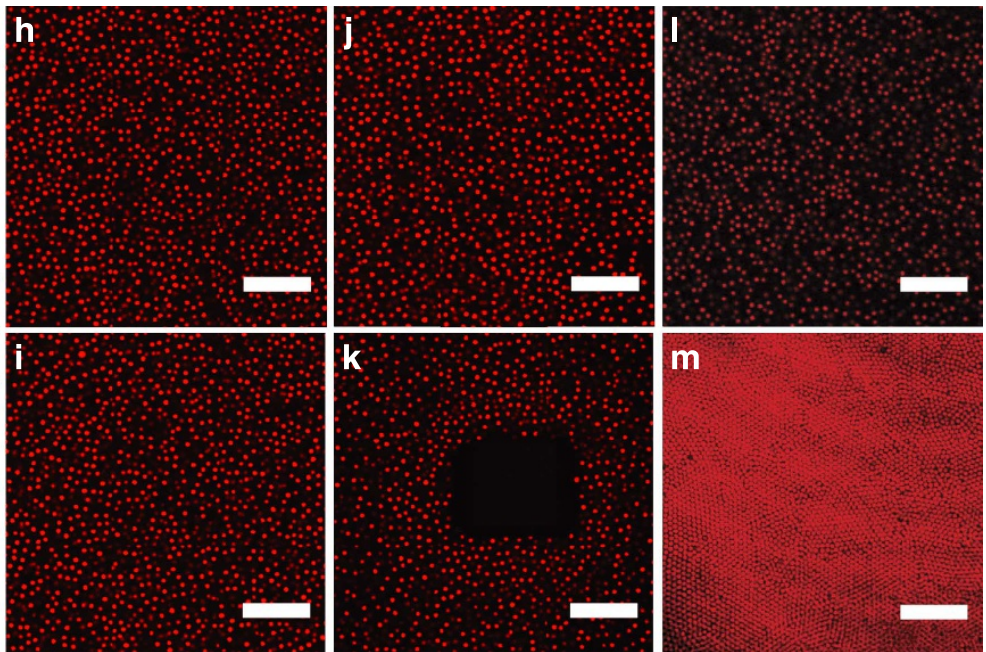

m

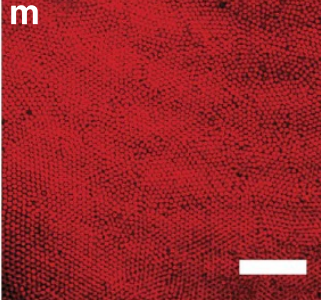

Figure 1 | Photo-induced colloidal assembly phenomenon. (a) PHSA-stabilized PMMA colloids $(1.4 \mu \mathrm{m})$ at an initial volume fraction of 0.05 are initially dispersed in solution in contact with the ITO-coated glass substrate. (b) A $488 \mathrm{~nm}$ laser or UV light source is focused at the objective and scanned across a region of interest. (c) Particle accumulation is observed at the illuminated region. (d-m) Each image pair shows the colloidal structure before and after $60 \mathrm{~s}$ of excitation at $488 \mathrm{~nm}$ with power density $12 \mathrm{~W} \mathrm{~cm}^{-2}(\mathbf{d}-\mathbf{k})$ and excitation at $360 \mathrm{~nm}$ with power density $0.62 \mathrm{~W} \mathrm{~cm}^{-2}(\mathbf{I}, \mathbf{m})$. The solvent is $\mathrm{CHB} /$ decalin with $50 \mu \mathrm{M}$ TBAB added $(\mathbf{d}-\mathbf{i}, \mathbf{I}, \mathbf{m})$ or no added TBAB (j, $\mathbf{k})$. In images $(\mathbf{d}, \mathbf{e}, \mathbf{j}, \mathbf{k}-\mathbf{m})$, the solution is in contact with an ITO-coated substrate; $(\mathbf{f}, \mathbf{g})$ the solution is in contact with a gold coated substrate; $\mathbf{h}, \mathbf{i}$ is a control experiment in which the solution is in contact with a pure silica substrate. Scale bars, $20 \mu \mathrm{m}$. 
sufficient for colloidal crystal ordering (Fig. 1e). The greatest concentration of particles was found within the illuminated region, with modest photobleaching (Fig. 1e). We know that the effect of the light on the self-assembly reported in Fig. 1 is not due to optical trapping and radiation pressure, because the particles are nearly refractive index matched with the solvent $(\Delta n / n=0.002)$. The observed colloidal velocity is three orders of magnitude greater than what would be expected due to optical trapping and radiation pressure, as per a calculation using the Mie scattering theory ${ }^{17,24}$.

The particle accumulation effect only occurred for the case of conductive or semi-conductive substrates. For example, replacing the ITO coating with gold still yielded particle accumulation (Fig. 1f,g). The corresponding control experiment, with illumination of a (uncoated) silica coverslip, yielded no particle accumulation (Fig. 1h,i). We also found that the effect requires direct contact of the ITO substrate with the colloidal solution. Specifically, when the ITO-coated substrate is flipped so that the silica side is in contact with the solution, or when the ITO layer is covered with a thin layer of deposited silica, the photo-induced motion is no longer observed. These observations, along with finite difference simulation that indicates very low temperature gradients in the system, rule out electrothermal and thermophoretic explanations of the photo-induced motion ${ }^{4,25}$.

The assembly was also a sensitive function of TBAB concentration. Figure $1 \mathrm{j}, \mathrm{k}$ shows that colloids were expelled from the illumination region, instead of collected, when the $50 \mu \mathrm{M}$ of $\mathrm{TBAB}$ was not added to the $\mathrm{CHB} /$ decalin solvent mixture. Particle accumulation was also observed at other illumination wavelengths. Illumination with a mercury metal halide lamp $(360 \mathrm{~nm})$ yielded accumulation of particles as indicated by Fig. 1l,m. Thus, the directed assembly phenomenon is common and predicated on the coupling of electromagnetic illumination to an absorptive, conductive substrate such as ITO. Additional features of the effect, such as the solvent conditions, the particle types and the illumination wavelengths in which assembly or depletion was observed, are available.

First, there is a strong effect of solvent conditions on whether or not the photo-induced effect is observed. While organic, low-dielectric constant systems, such as CHB and decalin, demonstrated significant photo-induced assembly response to electromagnetic illumination, systems of higher dielectric constants, such as dimethyl sulphoxide (DMSO) and water, showed little to no photo-induced effect. The role of solvent dielectric constant in the self-assembly of the colloids studied here has been previously established ${ }^{22}$, and the present effect is consistent with those previous observations.

Second, in solvents in which particles do display the photoinduced effect, whether or not assembly or depletion is observed is a strong function of the kind of colloids used. The photoinduced effect was studied for PMMA colloids with various surface stabilizers and polystyrene (PS) colloids. Assembly was observed under a variety of conditions for PMMA colloids. For example, assembly occurred for different substrates illuminated (gold and ITO coating), varying wavelengths of the laser (458, 476 , and $488 \mathrm{~nm}$ ), laser powers ranging from 2.7 to $12 \mathrm{~W} \mathrm{~cm}^{-2}$, initial volume fractions of particles varying from 0.0125 to 0.20 and particle sizes from 0.6 to $1.4 \mu \mathrm{m}$. All of these experiments were carried out with PHSA-stabilized PMMA colloids in $\mathrm{CHB} /$ decalin with a $1 \mathrm{mM} \mathrm{TBAB}$ electrolyte. Other surfacefunctionalized PMMA particles, such as those synthesized with the stabilizers polydimethylsiloxane (PDMS ${ }^{26}$ and diphenyldimethylsiloxane (DPDMS) ${ }^{27}$, showed depletion at the illuminated region, similar to that observed for PHSA-stabilized PMMA colloids with no TBAB. PS particles demonstrated no signs of assembly in both water and DMSO solvents of $0.1 \mathrm{mM}$
TBAB under visible light excitation, whereas, under UV illumination, PS particles were found to accumulate in DMSO.

Third, for particles and solvents that show the photo-induced effect, the magnitude of the effect, as indicated by the speed of the assembly and the extent and quality of the ordered structures formed, is a function of whether visible or UV light is used. With the UV illumination $(360 \mathrm{~nm})$, faster accumulation was observed relative to the case of visible light. Faster accumulations with UV illumination were observed for both PHSA-stabilized PMMA colloids and for PS particle suspensions. For these cases, PMMA particle suspensions were prepared in a $1 \mathrm{mM}$ TBAB solution of $\mathrm{CHB} /$ decalin.

Three-dimensional structure of the assembled crystals. The photo-induced effect yields finite-thickness quasi-2D colloidal crystals. At steady-state, and at the laser power densities studied, the penetration depth of the accumulation was about $10 \mu \mathrm{m}$, resulting in a stacking of about five crystalline layers (Fig. 2a,b).
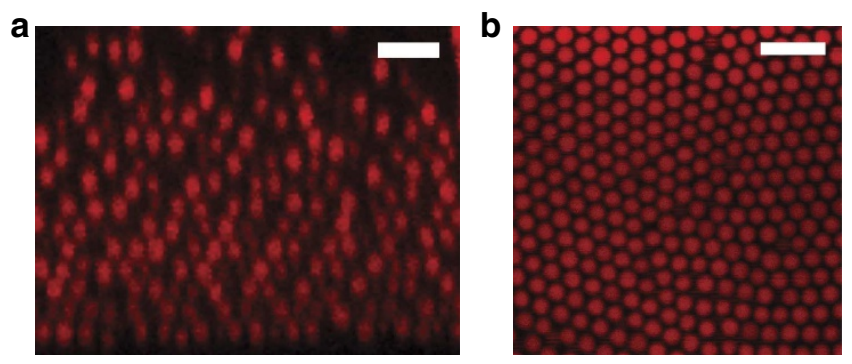

C
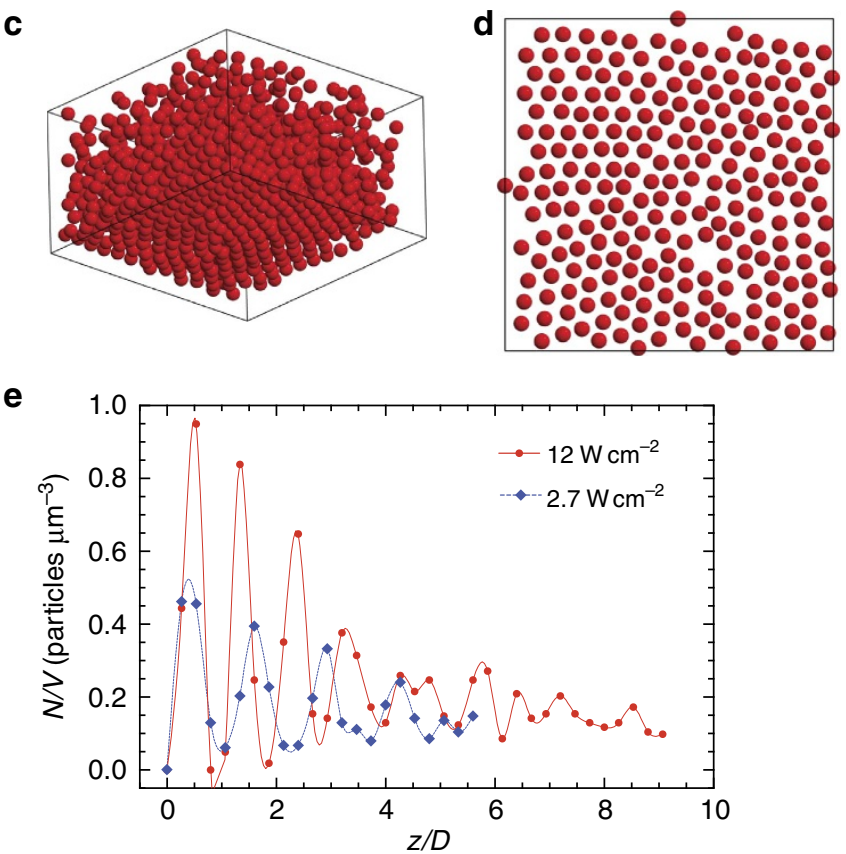

Figure 2 | Three-dimensional structure of the photo-induced assembly. The particle and solvent conditions are as in Fig. 1e and the illumination time is $120 \mathrm{~s}$. (a) Image acquired perpendicular to the ITO coverslip shows accumulation of multiple layers of colloids above the region of illumination. Scale bar, $5 \mu \mathrm{m}$. (b) Image of the same structure acquired at the ITO coverslip. Scale bar, $5 \mu \mathrm{m}$. (c) 3D rendering of CLSM-derived colloid centroids shows crystalline structure; colloid centroids were identified by image processing; (d) Rendering of the crystalline layer closest to the coverslip, which shows hexagonal ordering, with some defect structure. (e) Particle number density (N/V) of the assembly is a function of distance from the substrate and laser power density. 
The 3D structure of the crystal, as rendered by image analysis of CLSM volumes, showed close-packed ordering with some defect structure (Fig. 2c,d). The local number density $(N / V)$ of colloids was as large as 0.95 particles $\mu \mathrm{m}^{-3}$ at the ITO substrate at the largest power density studied $\left(12 \mathrm{~W} \mathrm{~cm}^{-2}\right)$. The periodic variation in particle density apparent in Fig. 2e is further evidence of the layered structure, consistent with a colloidal crystal. The effect of the light on the self-assembly is significant. As the power is changed from 12 to $2.7 \mathrm{~W} \mathrm{~cm}^{-2}$, the near-wall particle number density of the sample decreases from 0.95 to 0.46 particles $\mu \mathrm{m}^{-3}$. Both self-assemblies, however, have crystalline structure. Under these conditions, the near-wall crystalline volume fractions (within the first three layers) are 47 and 34\%, respectively. These values are consistent with that of the disorder to order transition boundary expected for the system of charged PHSA-stabilized PMMA colloids. The theoretical phase transition boundary was determined to be $\phi=0.36$, given the measured Debye screening length $(95 \mathrm{~nm})$ and the colloid zeta potential $(-9 \mathrm{mV})^{28}$.

Kinetics of the photo-induced assembly. The photo-induced assembly process occurs rapidly. The characteristic time for assembly is about $60 \mathrm{~s}$ (Fig. 3a-f). On laser exposure, particles are displaced from the suspension into the illuminated region, as also shown in Supplementary Movie 1. Particles begin to concentrate a few seconds after the ITO-coated surface is exposed to the laser (Fig. 3b), until a crystalline structure is formed. Accumulated particles display a close-packed crystal ordering (Fig. 3e). For initial volume fractions studied $\left(\phi_{0}=0.0125-0.20\right)$, the photoinduced volume fraction monotonically increases with time and reaches a steady state (Fig. $3 g$ ). From Fig. $3 g$, a characteristic time for crystallization, $t_{c}$, was defined as the time for the assembly to reach $99 \%$ of its steady-state volume fraction. Figure 3 h shows that $t_{\mathrm{c}}$ decreases with increasing $\phi_{0}$ and laser power.

The sedimentation dynamics was used to infer a particle velocity from measurements of $t_{c}$, as shown in Supplementary Fig. 1. The photo-induced particle velocity $(1,000 \mu \mathrm{M}$ TBAB and $12 \mathrm{~W} \mathrm{~cm}^{-2}$ ) was found to be $0.28 \pm 0.02 \mu \mathrm{m} \mathrm{s}^{-1}$, independent of the initial volume fraction. The decrease in $t_{c}$ with $\phi_{0}$ is a consequence of the faster filling of the assembly region due to the greater local availability of colloids at higher $\phi_{0}$. That is, at low $\phi_{0}$, it takes longer to crystallize the near-wall layer because the accumulating colloids must be drawn from a larger reservoir.
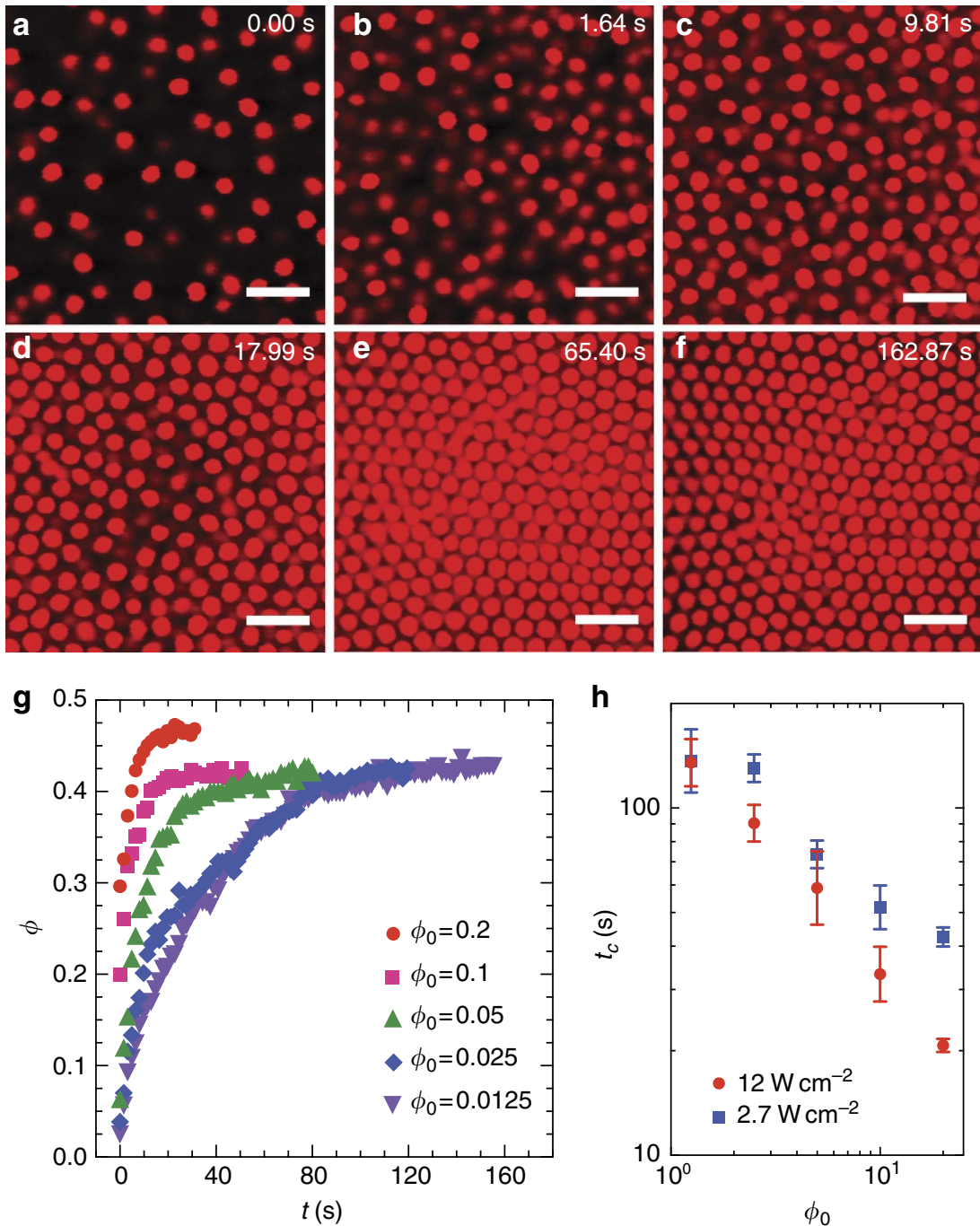

Figure 3 | Kinetics of assembly under optical field excitation. (a-f) Time lapse images of PMMA particles $\left(\phi_{0}=0.05\right)$ concentrating in the $25 \times 25 \mu m^{2}$ region that was illuminated. The particle and solvent conditions are as in Fig. 1e. Scale bar, $5 \mu \mathrm{m}$. (g) Particle volume fraction, $\phi$, monotonically increases until it reaches steady state. (h) Quantification of the critical concentration time, $t_{c}$, shows that time for accumulation decreases with increasing laser power density and initial volume fraction. The error bars indicate s.d. 
Effect of TBAB concentration on the photo-induced assembly. The effect of light on assembly was a strong function of TBAB concentration. Figure 4 shows a transition from depletion to assembly with varying [TBAB]. As TBAB concentration is varied from 0 to $1,000 \mu \mathrm{M}$, reversal of the photo-induced effect occurs abruptly between TBAB concentrations of 7.5 and $8.75 \mu \mathrm{M}$. Below the threshold condition of $7.5 \mu \mathrm{M}$, depletion of particles from the illuminated region was observed (Fig. $4 a-c$ ). The kinetics of the depletion effect are in Supplementary Movie 2. Between salt conditions of 8.75 and $10 \mu \mathrm{M}$, the system demonstrated a weak driving force for assembly, as evidenced by the observation that the accumulation effect was not strong enough to generate a colloidal crystal. A disordered, liquid structure was instead observed (Fig. 4d). For TBAB concentrations greater than $20 \mu \mathrm{M}$, the steady-state assemblies generated were crystalline. Increase in salt concentrations led to a small increase in assembly volume fraction in this range of TBAB concentration. This small change is due to the crystalline structure having smaller particle separations at higher salt concentrations, due to the changing Debye length.

The effect of TBAB on the photo-induced assembly was quantified by measuring the local, near-wall colloid volume fraction as a function of time. Consistent with qualitative observations, $\phi$ decreases relative to the initial volume fraction for $[\mathrm{TBAB}]<7.5 \mu \mathrm{M}$ and increases for $[\mathrm{TBAB}]>8.75 \mu \mathrm{M}$ (Fig. 4g). Near steady-state volume fractions were reached for all conditions. By plotting the final volume fraction measured at each $\mathrm{TBAB}$ condition, the abrupt transition from colloidal depletion (at low TBAB concentration) to assembly (at high TBAB concentration) is apparent (Fig. 4h). The abruptness of the change, and the fact that it occurs at a finite TBAB concentration, argues against a role of the changing Debye length in this transition. Rather, we will show that the abrupt change at $\sim 8 \mu \mathrm{M}$ TBAB is more likely due to the effect of [TBAB] on the colloid zeta potential.

Taken together, these results constrain possible mechanisms of the photo-induced assembly. To summarize, the mechanism must be consistent with local colloid motion of $\sim 0.3 \mu \mathrm{m} \mathrm{s}^{-1}$ induced when a metallic or semiconducting substrate is illuminated with a $488 \mathrm{~nm}$ visible light at a power of $12 \mathrm{~W} \mathrm{~cm}^{-2}$ (for the UV case, the corresponding measured velocity is $1.8 \pm 0.3 \mu \mathrm{ms}^{-1}$ at a power of $0.62 \mathrm{~W} \mathrm{~cm}^{-2}$ ); near refractive index matching $(\Delta n / n=0.002)$ of the colloids and solvent; requirement that the semiconducting or metallic substrate is in direct contact with the colloidal solution; change from colloidal depletion to assembly in the region of illumination at $\sim 8 \mu \mathrm{M}$ as $\mathrm{TBAB}$ concentration is varied between 0 and $1,000 \mu \mathrm{M}$.

Mechanism of the photo-induced colloidal crystallization. Figure 5 suggests a mechanism consistent with these findings. Independent measurements of the zeta potential of the PMMA colloids (Fig. 5a) show a remarkable coincidence between the sign of the measured colloid zeta potential (which reverses at $\sim 20 \mu \mathrm{M}$ TBAB) and the switch of the effect from depletion to assembly (which occurs at $\sim 8 \mu \mathrm{M}$ TBAB). This sensitivity of PMMA
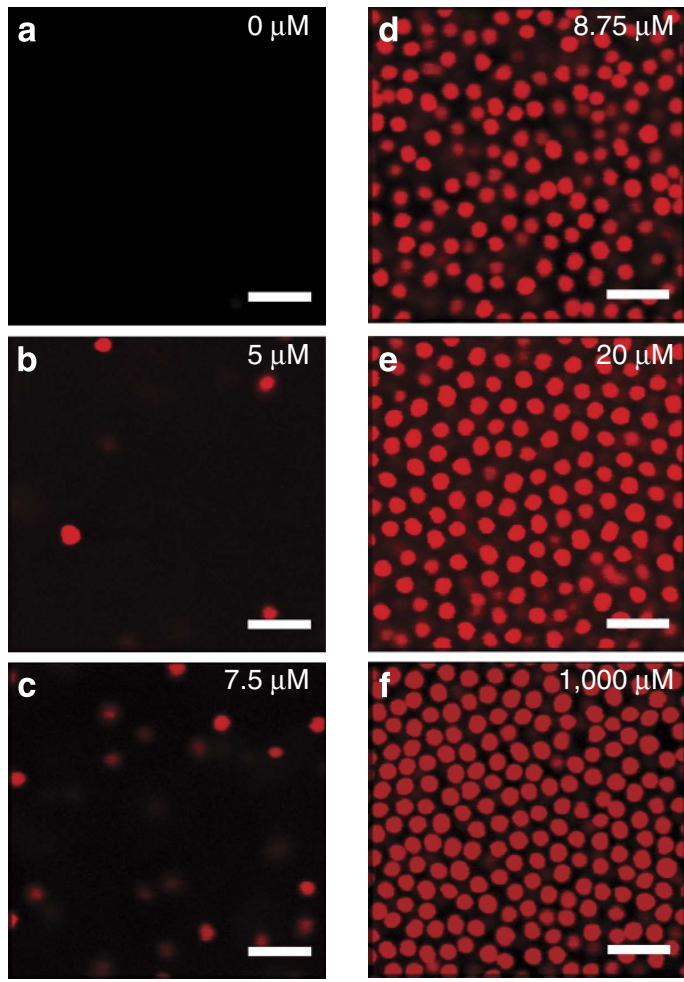

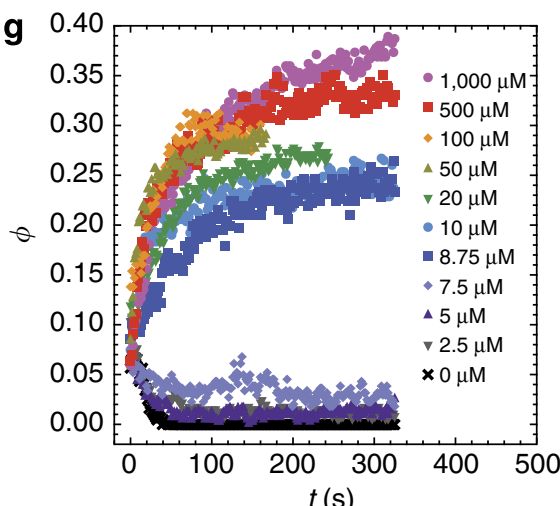

h

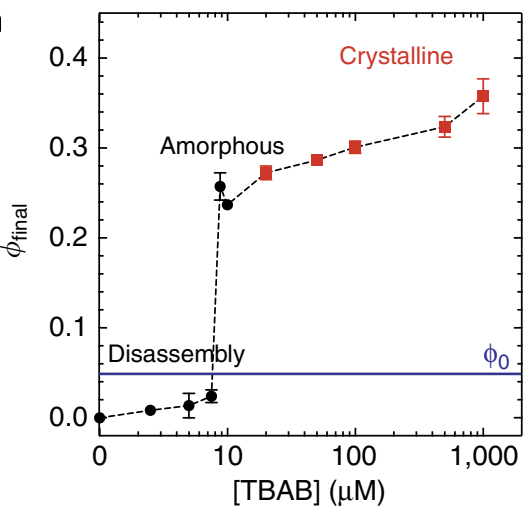

Figure 4 | Influence of the TBAB concentration on the photo-induced effect. Images are acquired $150 \mathrm{~s}$ after illumination and the initial colloid volume fraction is 0.05 . For these colloids, the particle zeta potential is a known function of TBAB concentration. (a-c) Images show PMMA colloid migration away from the illuminated region, thereby generating a void region of low colloid concentration. (d-f) Images show PMMA colloid migration towards the illuminated region, thereby leading to concentration and assembly. Scale bar, $5 \mu \mathrm{m}$. (g) For TBAB concentrations under $7.5 \mu \mathrm{M}, \phi$ decreases from its initial volume fraction, indicating depletion of particles within the region of interest. Above $7.5 \mu \mathrm{M} \mathrm{TBAB}$, the volume fraction increases monotonically to a higher final volume fraction. (h) Final volume fraction values are extracted from $\mathbf{g}$ and plotted against their TBAB concentrations. There is a distinct transition between 7.5 and $8.75 \mu \mathrm{M} \mathrm{TBAB}$; depletion is observed below this transition and assembly is observed above. The error bars indicate s.d. 

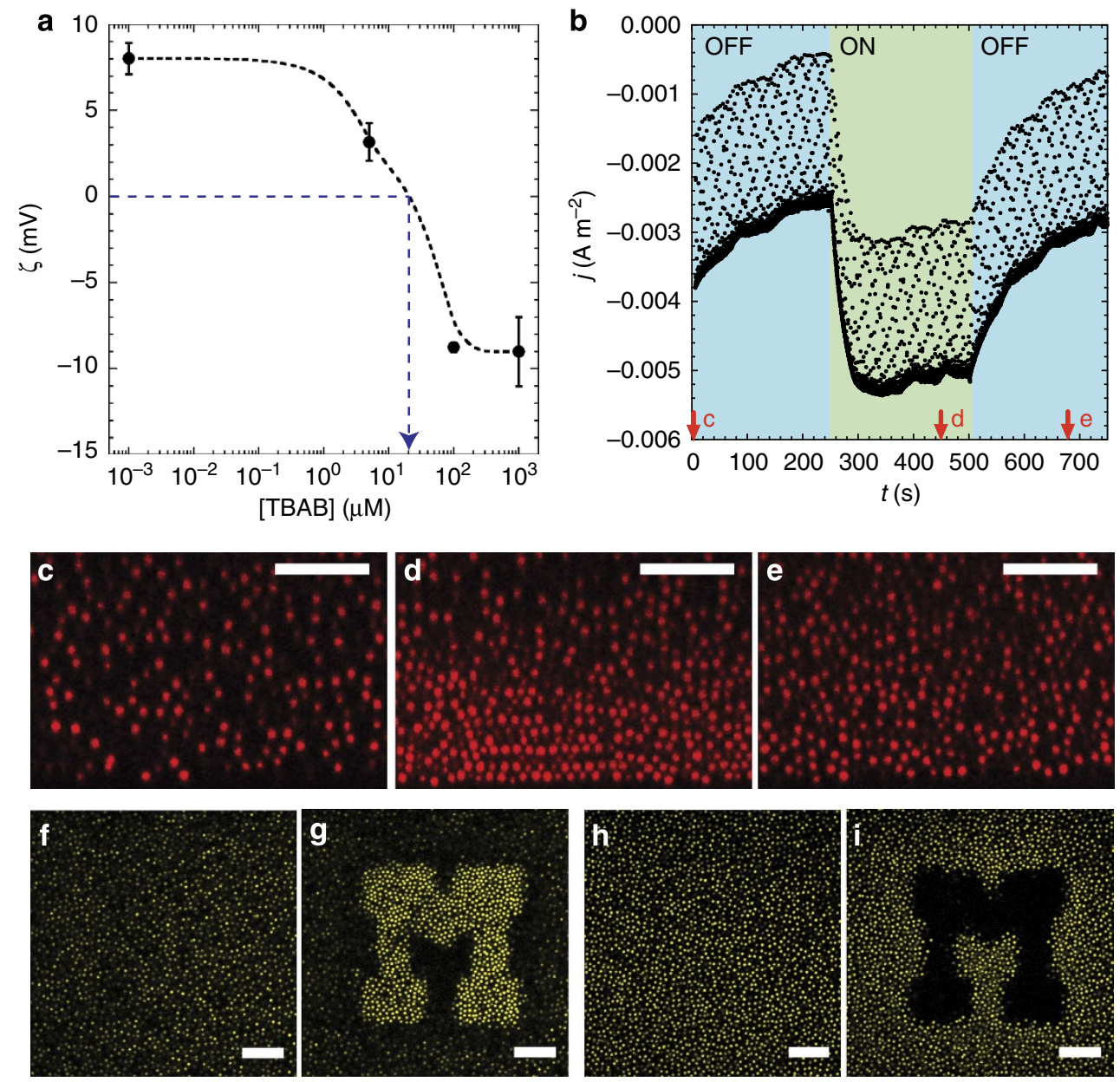

Figure 5 | Electrophoretic origin of the photo-induced assembly mechanism. (a) Zeta potential values of PHSA-stabilized PMMA colloids undergo a change in sign at about $20 \mu \mathrm{M}$ added TBAB. The dotted curve is a line to guide the eye. The error bars indicate s.d. (b) When the photo-induced effect is generated in a cell with electrodes, the measured current exhibits a rapid change as a direct response of the UV light being turned on and off. This experiment was carried out with PHSA-stabilized PMMA colloids prepared at $5 \%$ volume fraction and 1,000 $\mu$ M concentration of TBAB. (c-e) CLSM images corresponding to the times marked in $\mathbf{b}$. Images taken perpendicular to the coverslip show that the effect of light on the particle assembly is temporally correlated with changes in current density due to the illumination. Scale bar, $15 \mu \mathrm{m}$. (f-i) Because the current density is produced only in the illuminated region, the photo-induced assembly and depletion effects can be achieved when complex shapes are illuminated by the light, as is possible by, for example, using the optics of the scanning microscope. $(\mathbf{f}, \mathbf{g})$ Before and after images of colloidal assembly show accumulation of particles in the illuminated region of complex shape. The colloidal solution was prepared at $5 \%$ initial volume fraction with $1,000 \mu \mathrm{M}$ TBAB in CHB/decalin. (h,i) Before and after images of colloidal depletion show depletion of particles within the illuminated region of complex shape. The initial colloidal volume fraction was $10 \%$, and the solvent was pure $\mathrm{CHB} /$ decalin. Scale bars, $20 \mu \mathrm{m}$.

colloid zeta potential to TBAB concentration, including a change in the sign of the zeta potential, is consistent with the literature ${ }^{29}$. The coincidence of the assembly/depletion transition and the change in sign of the zeta-potential suggest that the photoinduced assembly effect is induced by light-induced electrophoresis of the colloids. This mechanism would imply the existence of an electric field in the system. While no electrode geometry has been fabricated into the system, the metallic surface itself is potentially reactive ${ }^{3,30}$. If the surface were a substrate for chemical reactions, the resulting depletion in chemical species at the substrate would result in an ion flow normal to the illuminated region of the substrate. This ion flow will produce a current that yields an electric field of magnitude $E=\frac{j}{\lambda_{0}}$, where $j$ is the measured current density (per illuminated area) and $\lambda_{0}$ is the conductivity of the solution ${ }^{31}$. Thus, if photo-induced electrophoresis is the mechanism for the observed directed assembly, then a current ought to be set up in the system when light illuminates the ITO substrate. The current itself is quite small because of the small illumination area $\left(\sim 1.8 \times 10^{4} \mu \mathrm{m}^{2}\right.$ in most cases). The magnitude and direction of the current should be consistent with the magnitude and sign of the colloid zetapotential. Ion flows of the kind hypothesized here have previously been reported for non-aqueous solvents ${ }^{32}$.

To test this hypothesis, we performed the following experiment. For a solution of 5 vol\% particles in $\mathrm{CHB} /$ decalin with $1,000 \mu \mathrm{M}$ TBAB, the current density, $j$, was measured as UV illumination $\left(360 \mathrm{~nm}, 0.62 \mathrm{~W} \mathrm{~cm}^{-2}\right)$ was turned on and off (Fig. 5b). We found a significant change in the current density as the UV light was switched on and off. Simultaneously, CLSM images were collected (Fig. $5 \mathrm{c}-\mathrm{e}$ ). The results show that the application of light coincides with both change in the current density and the observation of assembly. Additional details are also consistent with the mechanism: First, the negative zeta potential (at 1,000 $\mu \mathrm{M} \mathrm{TBAB}$ ) is in accord with the observation of colloidal motion towards the illuminated electrode at this condition. Specifically, particle motion in this direction indicates 
that the illuminated electrode is the anode, consistent with the negative sign of the measured cathodic current, as plotted in Fig. 5b (ref. 31). Second, the up and down motion of the microscope's optical scanner is observable in the current density (Fig. 5b), consistent with an effect of visible light on accumulation. Third, the magnitude of the colloidal velocity predicted from the electric field is consistent with direct measurements of UV-induced velocities. Further details of these observations are given in Supplementary Note 1.

\section{Discussion}

The photo-induced assembly, therefore, occurs due to an electric field generated by a light-induced current. Similar types of photoinduced current modulation have been observed in the past ${ }^{3}$, but never in the absence of an externally applied electric field and fixed electrodes. Here, instead, the electric field is generated internally. It is this internally generated field, induced by light alone, that allows the simultaneous spatial and temporal control of the assembly without need for other hardware, such as templating or electrodes. We point to two potential origins of the light-induced chemistry. The light could catalyse a reaction of dissolved species, such as residual water, oxygen, or surfactants such as TBAB at the ITO substrate; these redox reactions would drive a current that would induce an electric field; or, the light-induced ionization of dissolved species, such as TBAB, could create a concentration gradient within the solution, spontaneously setting up an electric field and thereby generating electrodiffusiophoresis ${ }^{33}$. Both mechanisms are consistent with the observation that the photo-induced motion requires illumination of a semiconducting or metallic substrate that is in direct contact with the colloidal solution.

The fact that the phenomenon requires a light/reactive substrate interaction means that the effect is extremely localized in space and time. Figure 5f-i shows application of this understanding to create complex shapes of colloids. A $405 \mathrm{~nm}$ laser scanned this intricate design, drawn as a region-ofinterest (ROI) by using the microscope's optical scanner. The experimental conditions of the assembly (Fig. 5f,g) and the depletion (Fig. 5h,i) are in the figure legend. The kinetics of these reconfigurable colloidal assemblies are available in Supplementary Movies 3,4. We find that colloids rapidly assemble and deplete within a few seconds of illumination to form structures with sharp features and well-defined boundaries. The definition of these features and boundaries are as good for the case of depletion as for assembly. Both kinds of structures created are fully reversible. The results indicate the method's rapidity, reversibility and spatial specificity for producing 3D regions of colloidal ordering and depletion. With these characteristics, the photoinduced electrophoretic method has broad application for new materials comprising reconfigurable assemblies, especially for the template free, spatially selective assembly of 3D colloidal crystals.

\footnotetext{
Methods

Colloidal system and sample preparation. The colloidal particles used for the photo-induced assembly were PHSA-stabilized PMMA $(D=1.40 \mu \mathrm{m}, 5.6 \%$ polydispersity). Particles were dispersed in a refractive index- and density-matched solvent mixture of $66 \mathrm{vol} \%$ cyclohexyl-bromide (CHB) and $34 \mathrm{vol} \%$ decahydronaphthalene (decalin). Experiments were carried out in suspensions of initial volume fraction, $\phi_{0}$, varying from 0.0125 to 0.20 and added TBAB concentration varying from 0 to $1,000 \mu \mathrm{M}$.

Two hundred microlitres of colloidal suspension was dispensed into a specimen vial with a cylindrical geometry $(D=10 \mathrm{~mm}, H=1.25 \mathrm{~mm})$. ITO-coated microscope coverslips $(d \sim 0.170 \mathrm{~mm})$ were used as the bottom surface of the vial, with the ITO coating facing inwards. The ITO coating was $\sim 65 \mathrm{~nm}$ thick with $\sim 500 \Omega$ sq. sheet resistance ${ }^{-1}$ (ZC \& R Coatings, Inc.). In one set of experiments, a gold coating was used instead of the ITO coating $(\sim 15 \mathrm{~nm}$ thick with $\sim 7.5 \mathrm{~nm}$ chrome
} layer, Denton Vacuum).
Light illumination. Two different light sources were used for the photo-induced assembly. First, $488 \mathrm{~nm}$ laser light was focused at the ITO-coated coverslip using a $100 \mathrm{X}$ oil immersion objective lens $(N A=1.40)$. The laser power was delivered by using a confocal laser scanning microscope (CLSM, Leica TCS SP2 or Nikon A1R), in which a pointwise scan of the region $\left(25 \times 25 \mu \mathrm{m}^{2}\right)$ was performed with a duty cycle of $66 \%$. The laser power density varied from 2.7 to $12 \mathrm{~W} \mathrm{~cm}^{-2}$ (Optical power meter 1916-C, detector 818-ST, Newport). Second, a UV light source (Leica EL6000, Mercury metal halide bulb, $360 \mathrm{~nm}$ ) was used for illumination in some cases. This radiation was projected onto a circular region $(D=150 \mu \mathrm{m})$ with a mean flux of $0.62 \mathrm{~W} \mathrm{~cm}^{-2}$ (UV meter 306, OAI). Another UV light source (Nikon A1R stimulation scan, $405 \mathrm{~nm}$ ) was used to scan a ROI of a complex shape.

Confocal microscope imaging conditions. CLSM was used to characterize the kinetics and structure of the photo-induced assembly (100X objective, $N A=1.40)$. For the assembly kinetics, time series of $100-150$ frames $(512 \times 512$ pixels $)$ with spatial resolution of $49 \mathrm{~nm}$ pixel $^{-1}$ were collected at a frame rate of $1.6 \mathrm{~s}$. For the assembly structure, 3D image volumes of $25 \times 25 \times 10 \mu \mathrm{m}^{3}$ were acquired at the voxel size of $49 \times 49 \times 46 \mathrm{~nm}^{3}$. Centroidal coordinates of all particles were determined by methods discussed in Crocker et al. and characterized in Dibble et al. ${ }^{34,35}$ The image analysis yielded centroidal coordinates resolved at the scale of $\pm 35 \mathrm{~nm}$ in the object plane and $\pm 45 \mathrm{~nm}$ in the axial direction. From these centroidal coordinates, we characterized the crystallization kinetics by determining the number of particles identified at each time frame. The structure of the ordered array was visualized by rendering the centroidal coordinates. The particle number density as a function of height above the ITO-coated substrate was determined by evaluating the number of particles in slices of size $21 \times 21 \times 0.4 \mu \mathrm{m}^{3}$. The particle volume fractions of the crystals were calculated from the three layers closest to the coverslip. For the analysis of Figs 3,4, the near-wall volume fraction was that of the close-packed layer at the coverslip.

The ROI stimulation method of CLSM (Nikon A1R, $100 \times$ oil objective, $N A=1.49$ ) was used to create assembly and depletion structures of complex shapes. Simultaneous imaging $(561.6 \mathrm{~nm})$ and scanning $(405 \mathrm{~nm})$ were done with a resonant scanner at a frame rate of $0.067 \mathrm{~s}$. Stimulation area was defined by the ROI designed as a specific shape on the confocal software. Images $(512 \times 512$ pixels $)$ were collected at a spatial resolution of $0.249 \mu \mathrm{mpixel}^{-1}$.

Photo-induced velocity measurements. The laser-induced colloidal velocity was calculated by adapting an analysis available for sedimentation kinetics ${ }^{28}$. The details of the analysis method are further described in Supplementary Note 2. The UV-induced colloidal velocity was measured from tracking the colloidal motion. Here, the distance that the particle travelled within two consecutive images was measured and divided by the frame rate $(0.815 \mathrm{~s})$ to calculate the particle velocity. From a series of $\sim 200$ images, sequential images were extracted and analysed to determine the average velocity.

Zeta potential and current measurements. Zeta potential and current measurements were made in the DC electric field device described by Shah $e t$ al. $^{32}$ Zeta-potential measurements were carried out in $1.25 \mathrm{vol} \%$ particle suspensions with TBAB concentrations varying from 0 to $1,000 \mu \mathrm{M}$. Particle mobility was tracked in planes perpendicular to the coverslip as a constant current was applied with the potentiostat (Autolab PGSTAT 128N) across the solution. Images were collected at $0.815 \mathrm{~s}$ per frame. Given the measured mobility, the electric field set up by the applied current, solvent properties $(\varepsilon=5.962, \mu=0.0025 \mathrm{Pas})$ and the electrolyte properties $\left(\Lambda_{\mathrm{TBA}+}^{0}=6.5 \mathrm{~cm}^{2} \mathrm{~S} \mathrm{~mol}^{-1} \text { and } \Lambda_{\mathrm{Br}-}^{0}=14.5 \mathrm{~cm}^{2} \mathrm{~S} \mathrm{~mol}^{-1}\right)^{29}$ zeta-potentials were computed by using the method of O'Brien and White ${ }^{36}$. Similarly the current density changes within the suspension were measured as light was turned on and off. UV illumination ( $360 \mathrm{~nm}$ peak wavelength) at $0.62 \mathrm{~W} \mathrm{~cm}^{-2}$ was applied. The potentiostat was used to measure the current density variations under a constant zero potential (current detection range $\sim 100 \mathrm{pA}$ ).

\section{References}

1. Grier, D. G. A revolution in optical manipulation. Nature 424, 810-816 (2003).

2. Vossen, D. L. J., van der Horst, A., Dogterom, M. \& van Blaaderen, A. Optical tweezers and confocal microscopy for simultaneous three-dimensional manipulation and imaging in concentrated colloidal dispersions. Rev. Sci. Instrum. 75, 2960-2970 (2004).

3. Hayward, R. C., Saville, D. A. \& Aksay, I. A. Electrophoretic assembly of colloidal crystals with optically tunable micropatterns. Nature 404, 56-59 (2000).

4. Kumar, A. et al. Optically modulated electrokinetic manipulation and concentration of colloidal particles near an electrode surface. Langmuir 26, 5262-5272 (2010).

5. George, M. C. et al. Direct laser writing of photoresponsive colloids for microscale patterning of 3D porous structures. Adv. Mater. 21, 66-70 (2009)

6. Feng, L. et al. Cinnamate-based DNA photolithography. Nat. Mater. 12, 747-753 (2013).

7. Chronis, N., Liu, G., Jeong, K. \& Lee, L. Tunable liquid-filled microlens array integrated with microfluidic network. Opt. Express 11, 2370-2378 (2003) 
8. Holtz, J. H. \& Asher, S. A. Polymerized colloidal crystal hydrogel films as intelligent chemical sensing materials. Nature 389, 829-832 (1997).

9. Yang, J. J., Borghetti, J., Murphy, D., Stewart, D. R. \& Williams, R. S. A family of electronically reconfigurable nanodevices. Adv. Mater. 21, 3754-3758 (2009).

10. Velev, O. D., Tessier, P. M., Lenhoff, A. M. \& Kaler, E. W. Materials: a class of porous metallic nanostructures. Nature 401, 548-548 (1999).

11. Ma, F., Wu, D. T. \& Wu, N. Formation of colloidal molecules induced by alternating-current electric fields. J. Am. Chem. Soc. 135, 7839-7842 (2013).

12. Wu, N. \& Russel, W. B. Micro- and nano-patterns created via electrohydrodynamic instabilities. Nano Today 4, 180-192 (2009).

13. Grzelczak, M., Vermant, J., Furst, E. M. \& Liz-Marzán, L. M. Directed selfassembly of nanoparticles. ACS Nano 4, 3591-3605 (2010).

14. Lee, W., Chan, A., Bevan, M. A., Lewis, J. A. \& Braun, P. V. Nanoparticlemediated epitaxial assembly of colloidal crystals on patterned substrates. Langmuir 20, 5262-5270 (2004)

15. van Blaaderen, A., Ruel, R. \& Wiltzius, P. Template-directed colloidal crystallization. Nature 385, 321-324 (1997).

16. Fernandes, G. E., Beltran-Villegas, D. J. \& Bevan, M. A. Spatially controlled reversible colloidal self-assembly. J. Chem. Phys. 131, 134705 (2009).

17. Ashkin, A. Acceleration and trapping of particles by radiation pressure. Phys. Rev. Lett. 24, 156-159 (1970).

18. Srivastava, S. et al. Light-controlled self-assembly of semiconductor nanoparticles into twisted ribbons. Science 327, 1355-1359 (2010).

19. Klajn, R., Bishop, K. J. M. \& Grzybowski, B. A. Light-controlled self-assembly of reversible and irreversible nanoparticle suprastructures. Proc. Natl Acad. Sci. 104, 10305-10309 (2007).

20. Palacci, J., Sacanna, S., Steinberg, A. P., Pine, D. J. \& Chaikin, P. M. Living crystals of light-activated colloidal surfers. Science 339, 936-940 (2013).

21. Pusey, P. N. \& van Megen, W. Phase behaviour of concentrated suspensions of nearly hard colloidal spheres. Nature 320, 340-342 (1986).

22. Yethiraj, A. \& van Blaaderen, A. A colloidal model system with an interaction tunable from hard sphere to soft and dipolar. Nature 421, 513-517 (2003).

23. Leunissen, M. E. et al. Ionic colloidal crystals of oppositely charged particles. Nature 437, 235-240 (2005).

24. Seefeldt, K. F. \& Solomon, M. J. Self-diffusion in dilute colloidal suspensions with attractive potential interactions. Phys. Rev. E 67, 050402 (2003).

25. Duhr, S. \& Braun, D. Two-dimensional colloidal crystals formed by thermophoresis and convection. Appl. Phys. Lett. 86, 131921 (2005).

26. Klein, S. M., Manoharan, V. N., Pine, D. J. \& Lange, F. F. Preparation of monodisperse PMMA microspheres in nonpolar solvents by dispersion polymerization with a macromonomeric stabilizer. Colloid Polym. Sci. 282, 7-13 (2003).

27. Kogan, M., Dibble, C. J., Rogers, R. E. \& Solomon, M. J. Viscous solvent colloidal system for direct visualization of suspension structure, dynamics and rheology. J. Colloid. Interface Sci. 318, 252-263 (2008).
28. Russel, W. B., Saville, D. A. \& Schowalter, W. R. Colloidal Dispersions (Cambridge University Press, 1989).

29. Royall, C. P., Leunissen, M. E. \& van Blaaderen, A. A new colloidal model system to study long-range interactions quantitatively in real space. J. Phys. Condens. Matter 15, S3581 (2003).

30. van den Meerakker, J. E. A. M., Meulenkamp, E. A. \& Scholten, M. Photo)electrochemical characterization of tin-doped indium oxide. J. Appl. Phys. 74, 3282-3288 (1993).

31. Bard, A. J. \& Faulkner, L. R. Electrochemical Methods: Fundamentals and Applications (Wiley, 1980).

32. Shah, A. A. et al. Liquid crystal order in colloidal suspensions of spheroidal particles by direct current electric field assembly. Small 8, 1551-1562 (2012).

33. Ebel, J. P., Anderson, J. L. \& Prieve, D. C. Diffusiophoresis of latex particles in electrolyte gradients. Langmuir 4, 396-406 (1988).

34. Crocker, J. C. \& Grier, D. G. Methods of digital video microscopy for colloidal studies. J. Colloid. Interface Sci. 179, 298-310 (1996).

35. Dibble, C. J., Kogan, M. \& Solomon, M. J. Structure and dynamics of colloidal depletion gels: coincidence of transitions and heterogeneity. Phys. Rev. E 74, 041403 (2006).

36. O'Brien, R. W. \& White, L. R. Electrophoretic mobility of a spherical colloidal particle. J. Chem. Soc. Faraday Trans. 74, 1607-1626 (1978).

\section{Acknowledgements}

This work was supported by the National Science Foundation Graduate Research Fellowship program. A.A.S. and M.J.S. acknowledge the U.S. Army Research Office (Grant Award W911NF-10-1-0518). Any opinions, findings and conclusions or recommendations expressed in this publication are those of the authors and do not necessarily reflect the views of the DOD. We also thank Professor C.W. Monroe for the use of his potentiostat.

\section{Author contributions}

A.A.S. first discovered the phenomenon. Y.K. synthesized the colloidal particles and carried out the experiments. Y.K. and M.J.S. designed the project, analysed the data and wrote the manuscript. All authors discussed and commented on the manuscript.

\section{Additional information}

Supplementary Information accompanies this paper at http://www.nature.com/nature communications

Competing financial interests: The authors declare no competing financial interests.

Reprints and permission information is available online at http://npg.nature.com/ reprintsandpermissions/

How to cite this article: Kim, Y. et al. Spatially and temporally reconfigurable assembly of colloidal crystals. Nat. Commun. 5:3676 doi: 10.1038/ncomms4676 (2014). 